\title{
Stefan HEIDL, Philosophical Problems of Behavioural Economics
}

David Philippy

\section{OpenEdition}

Édition électronique

URL : https://journals.openedition.org/ress/3878

DOI : $10.4000 /$ ress.3878

ISSN : 1663-4446

Éditeur

Librairie Droz

\section{Édition imprimée}

Date de publication : 25 mai 2018

Pagination : 296-299

ISSN : 0048-8046

\section{Référence électronique}

David Philippy, «Stefan HEIDL, Philosophical Problems of Behavioural Economics », Revue européenne des sciences sociales [En ligne], 56-1 | 2018, mis en ligne le 10 novembre 2017, consulté le 08 janvier 2022. URL : http://journals.openedition.org/ress/3878 ; DOI : https://doi.org/10.4000/ress.3878

Ce document a été généré automatiquement le 8 janvier 2022.

(c) Librairie Droz 


\title{
Stefan HEIDL, Philosophical Problems of Behavioural Economics
}

\author{
David Philippy
}

\section{RÉFÉRENCE}

Stefan HEIDL, 2016, Philosophical Problems of Behavioural Economics, London, Routledge, $164 \mathrm{p}$.

1 Cet ouvrage est une version révisée de la thèse doctorale de son auteur et prend la forme d'une invitation à (re)découvrir et à reformuler les débats méthodologiques contemporains autour de l'économie comportementale.

2 La thèse principale du livre concerne la signification épistémologique de l'économie comportementale : l'existence de cette jeune sous-discipline de la science économique ne peut être raisonnablement comprise comme le seul résultat de l'interdisciplinarité entre économie et psychologie. Pour l'auteur, l'émergence de l'économie comportementale est la manifestation d'un processus plus général de «déidéalisation » de la théorie économique. L'idéalisation étant entendue comme "une simplification délibérée d'un objet complexe avec l'objectif de le rendre plus malléable» (p. 2, traduit par nous).

3 Aujourd'hui, il est en effet d'usage de présenter l'économie comportementale comme un programme de recherche issu des apports mutuels de l'économie et de la psychologie. La définition désormais canonique de Colin Camerer et George Loewenstein (2004) pose comme objectif central l'amélioration de la capacité prédictive des modèles grâce à des hypothèses comportementales plus réalistes. Ces nouveaux modèles permettraient à terme d'augmenter l'efficacité des politiques publiques.

4 Après quelques brefs rappels théoriques concernant l'économie comportementale, le chapitre introductif expose les insuffisances des relations inter-théoriques. Parmi ces dernières l'auteur distingue celles qui font appel au principe du réductionnisme théorique d'Ernest Nagel (The Structure of Science, New York, Harcourt, Brace \& World, 
1961). Ce type de réduction ne convient pas dans le cas de l'économie comportementale dans la mesure où il ne consiste vraisemblablement pas en une volonté de réduire la théorie économique à une théorie psychologique. L'auteur explore une variété d'autres possibilités de réduction potentiellement compatibles avec le cas de l'économie comportementale (fonctionnaliste, par mécanismes, etc.). Après avoir montré successivement les insuffisances de ces dernières, l'auteur propose la thèse de la « déidéalisation » de la théorie économique. L'objet du livre consiste à prouver le bienfondé de cette caractérisation.

5 Le chapitre 2 est consacré au statut ontologique des préférences en économie. L'auteur reprend la distinction entre les interprétations comportementaliste et mentaliste, cristallisée à la suite du débat entre "mindless economics " et "mindful economics" (Andrew Caplin et Andrew Schotter, The Foundations of Positive and Normative Economics : A Handbook, Oxford, Oxford University Press, 2008). La première renvoie aux "préférences révélées» de Paul A. Samuelson ("A note on the pure theory of consumer's behaviour », Economica, 5-17, 1938, p. 61-71) selon laquelle les préférences sont une donnée qu'il est possible d'observer au moment du choix. Dès lors, il est inutile de chercher à en savoir davantage sur l'origine de ces préférences. L'argumentation conduit à rejeter l'approche comportementaliste, en montrant qu'elle peut mener à l'indétermination des préférences de l'agent. De l'autre côté, l'approche mentaliste (adoptée par les économistes comportementaux) serait source d'hésitations sur le traitement des états mentaux subjectifs dans la mesure où ils ne sont pas observables, donc impossibles à tester. L'auteur fait appel à la méthode de confirmation $\mathrm{du}$ «bootstrap » de Clark Glymour (Theory and Evidence, Princeton, Princeton University Press, 1980) et montre au contraire de quelle manière l'approche mentaliste est capable de tester des hypothèses, montrant ainsi que ces inquiétudes sont injustifiées.

6 Dans le chapitre 3, l'auteur désamorce les attaques que l'approche dite "sélectionniste» pourrait émettre envers sa thèse. Contre l'économie comportementale, cette approche défend la théorie standard, sous prétexte que bien qu'incapable de donner une représentation fidèle du comportement des entreprises (et a fortiori des individus), elle est tout de même utile. L'argument repose sur le fait que le processus de sélection assurera que seules les entreprises conformes au modèle survivront sur le marché. Or, pour Stefan Heidl cette critique n'est pas recevable car son application est trop restrictive et son fonctionnement trop flou.

7 Le chapitre 4 montre comment les économistes comportementaux testent l'efficacité prédictive de la théorie économique standard. Dans les cas où cette dernière est erronée, une dé-idéalisation est nécessaire. Cette mise à l'épreuve de la théorie peut s'appuyer soit sur la modélisation, soit sur l'expérimentation. C'est grâce à ces deux outils qu'il est possible de départager les situations dans lesquelles la dé-idéalisation est nécessaire. Se focalisant sur les comportements sur le marché, l'auteur présente rapidement ces deux stratégies à travers plusieurs modèles (dont le modèle de choix aléatoire de Gary Becker par exemple) et quelques travaux expérimentaux (sur la diffusion des biais individuels au niveau du marché par exemple). L'objectif de ce chapitre est de présenter les outils de l'économie comportementale mais l'auteur ne justifie pas suffisamment ses choix. En effet, la comparaison proposée à la fin du chapitre entre ces deux outils est utile pour délimiter leur champ de compétence respectif mais reste trop générale sur leur faiblesse commune, à savoir l'extrapolation des résultats. 
8 Le chapitre 5 aborde la question centrale de l'indépendance explicative : la science économique devrait-elle se cantonner à l'explication de phénomènes particuliers dans des circonstances elles aussi particulières ? Et le cas échéant, comment articuler cette propriété à la définition de l'économie comportementale comme rencontre entre psychologie et économie? Pour les économistes comportementaux, la psychologie est un outil permettant d'enrichir leurs hypothèses pour augmenter la capacité prédictive des modèles. La dé-idéalisation apparaît alors comme un instrument explicatif permettant d'inclure des facteurs d'influence comportementale à la théorie standard. Ici, l'auteur pose l'incontournable question du rapport de l'économie comportementale au normatif, qu'il a le mérite de redéfinir avec pédagogie.

Le chapitre $6 \mathrm{~s}$ 'inscrit dans la continuité de la thèse de l'ouvrage : après avoir exposé les raisons pour lesquelles la thèse de la dé-idéalisation est recevable et montré comment les économistes comportementaux travaillent, l'auteur expose le procédé théorique qui consiste à localiser avec précision l'hypothèse qui doit être remise en cause. S'appuyant sur le concept de "localisation d'anomalie» de Lindley Darden (Theory Change in Science, New York, Oxford University Press, 1991), il présente les conditions dans lesquelles ces tests doivent être menés et souligne l'importance d'utiliser le contrôle expérimental pour éviter notamment l'écueil de la sous-détermination (on ne peut pas décider laquelle parmi plusieurs explications est la cause du phénomène observé). L'argument est brillamment présenté à l'aide de plusieurs exemples, dont le jeu de l'ultimatum et la théorie des perspectives. La confrontation d'un récit abstrait sur l'épistémologie à des travaux expérimentaux est plus que bienvenue et donne la mesure des implications de l'argument de l'auteur.

10 Dans les deux derniers chapitres, l'auteur achève de nous convaincre de la pertinence de sa thèse. Il démontre, à l'aide de deux cas (aversion à la perte et à l'iniquité) dans quels termes la dé-idéalisation prend concrètement forme dans le travail des économistes comportementaux. Contrairement au chapitre précédent, il ne s'agit pas ici d'évaluer la méthode employée, mais bien les résultats auxquels ils parviennent : la « structure » des théories comportementalistes, en ajoutant ou modifiant un paramètre dans le modèle, qui tiendrait compte d'une influence supplémentaire sur les préférences, permet de caractériser la dé-idéalisation. En effet, la connaissance attachée à l'économie comportementale s'agrège par itérations successives aux modèles, dans un mouvement de localisation-modification. L'auteur situe enfin l'économie comportementale par rapport à la théorie standard et à ses deux enjeux épistémologiques centraux : le rôle de la modélisation et le réalisme des hypothèses. L'un des points qui ressort en filigrane de cet ouvrage est que l'attachement des économistes comportementaux à la théorie du choix vise à expliquer les phénomènes au niveau agrégé. Pour l'auteur, cette quête de réalisme est plus que louable mais le type de modélisation reste le même que celui de la théorie standard. En somme, si les capacités prédictives des modèles sont effectivement accrues, les mêmes questions concernant le problème général de la modélisation en économie continuent à se poser dans les mêmes termes.

11 Cet ouvrage a l'immense mérite de remettre à plat les débats actuels en philosophie économique concernant cette nébuleuse qu'est l'économie comportementale. Il s'adresse avant tout aux lecteurs qui s'interrogent sur son statut, sa nature et ses méthodes. Si la thèse de la dé-idéalisation semble bien fonctionner ici, c'est peut-être parce que cette sous-discipline est encore jeune et qu'il semble aujourd'hui difficile de 
la caractériser autrement que comme un effort pour rapprocher le modèle de la réalité. La question du réalisme des hypothèses doit certes être prise au sérieux, et ce non seulement pour des raisons épistémologiques : l'idéalisation outrancière a fait gagner en scientificité ce qu'elle a fait perdre en sens à la discipline. Or, l'exigence de réalisme que le programme comportemental apporte avec lui semble appuyer cette reconquête de légitimité. L'ouvrage de Stefan Heidl le montre bien : l'économie comportementale fonctionne comme un engin de modification et d'ajout de paramètres dans les modèles standards. Mais dans ces conditions, quelle valeur donner à ce réalisme si nous ne donnons pas davantage de sens au modèle?

\section{AUTEURS}

\section{DAVID PHILIPPY}

Centre Walras-Pareto, Université de Lausanne 